\title{
Strictly Adhering to Infection Control Practices is the Key to Safe Surgical Procedures Amidst the COVID-19 Crisis
}

\author{
Rahul Garg ${ }^{(D)}$, Arghya Das (D) and Tuhina Banerjee* \\ Department of Microbiology, Institute of Medical Sciences, Banaras Hindu University, Varanasi - 221005 , India.
}

\begin{abstract}
Currently, the world is facing a challenge to curb the outbreak of the highly contagious COVID-19, which has significantly impacted many lives and is affecting the practice of healthcare professionals as well. During this containment phase, an influx of COVID-19 patients requiring surgical interventions is expected. To combat the situation, every health care staff must implement and adhere to various infection prevention and control measures. In this article, we have addressed the importance of basic infection prevention and control practices required for handling COVID-19 cases, especially, those requiring acute surgical care. On one hand, certain non-emergency procedures need to be delayed, on the other hand, all surgeries during this period need to be proactively performed with appropriate infection control precautions regardless of the COVID-19 status of the patient. Prudent decisions and a conservative approach while making the best choices regarding surgeries will not only ensure personal protection but will also preserve important resources, manpower, hospital beds, and equipment for repurposing during this urgent crisis.
\end{abstract}

Keywords: Surgery, COVID-19, Infection control, Operation, Preparedness

\footnotetext{
*Correspondence: drtuhina@yahoo.com

(Received: April 30, 2020; accepted: June 25, 2020)

Citation: Garg R, Das A, Banerjee T. Strictly Adhering to Infection Control Practices is the Key to Safe Surgical Procedures Amidst the COVID-19 Crisis. J Pure Appl Microbiol. 2020;14(2):1099-1105. doi: 10.22207/JPAM.14.2.03

(C) The Author(s) 2020. Open Access. This article is distributed under the terms of the Creative Commons Attribution 4.0 International License which permits unrestricted use, sharing, distribution, and reproduction in any medium, provided you give appropriate credit to the original author(s) and the source, provide a link to the Creative Commons license, and indicate if changes were made.
} 


\section{INTRODUCTION}

Currently, the world is facing a challenge to curb the outbreak of the novel coronavirus that was first reported in Wuhan, China on December 31, 2019 ${ }^{1}$. The World Health Organization (WHO) named the disease as "Corona Virus Disease 2019" (COVID-19) on February 11, 2020. With the help of next-generation sequencing, the genome sequence of the novel coronavirus was identified and subsequently named as the severe acute respiratory syndrome coronavirus 2 (SARS CoV- 2$)^{2}$. The rapid international spread of this highly contagious virus has caused concern worldwide, significantly impacting many lives, and affecting the practice of healthcare professionals as well. The dynamics of the infection transmission determine the response towards it and, prevention measures need to be taken appropriately. The infection control measures against SARS CoV-2 primarily focus on two modes of transmission viz. respiratory droplets and contact with contaminated surfaces in the form of fomites. Thus, in healthcare settings, it highlights the importance of basic infection prevention practices such as hand hygiene, appropriate use of personal protective equipment (PPE), and environmental cleaning. Additional airborne precautions such as the use of N95/ filter face piece 2 (FFP2)/ filter face piece 3 (FFP3) respirators are needed during aerosol-generating procedures (AGPS) performed on COVID-19 patients ${ }^{3}$.

Surgical care including both elective and emergency procedures are an important resource in any health system serving the community. With respect to the baseline rate of emergency operations in the medical facilities, there will be an influx of COVID-19 positive patients requiring surgical interventions during this outbreak. However, operating rooms (OR) are the high-risk areas for transmission of respiratory infections, and thus managing such patients becomes highly critical. Moreover, limited resources and understaffing greatly increase the risk of transmission and the burden on healthcare systems during this pandemic. Therefore, effort should be made to support the health care workers (HCWs) and maximize occupational safety, thus, improving overall patient care. To combat the present COVID-19 pandemic, implementation and adherence to infection prevention and control practices are of utmost importance for every health care staff. In this article, we have addressed the importance of basic infection prevention and control (IPC) practices required for the preparedness of handling COVID-19 cases, who require acute surgical care.

\section{Key considerations for the preparedness}

Administrative control is the first and most important step in any hospital preparedness towards the outbreak of any infectious disease. It consists of management measures that are expected to reduce the risk or exposure to health care staff ${ }^{4}$. A robust and dedicated team that should come into action immediately, include surgeons, anesthetists, members of the infection control team, operating room nursing staff, cleaning staff, security personnel, and the administrative staff. We propose a checklist to be considered for the preparedness for surgery during the COVID-19 pandemic (Table 1).

Hospital administration and surgeons should carefully review all scheduled elective procedures with a plan to postpone or cancel operations and other invasive procedures at least until the epidemiological curve flattens ${ }^{5}$. However, as the number of COVID-19 patients requiring care has been escalating over the last few months and they are expected to be on the rise for the next few weeks, the surgical care of patients should be limited to those with the imminent danger of losing their lives.

All suspected patients requiring surgery should be screened and should be considered as positive cases until proven otherwise. Thus, taking appropriate precautions will minimize the spread of the infection. The clinician should maintain a distance of $2 \mathrm{~m}$ from the patient while taking the patient's history which, in addition to routine case-related questions must also include a detailed epidemiological history. Moreover, thorough counseling of the relatives should be done before the surgery informing them about the hazards of the infection, if present and precautions to be taken during and after the procedure.

The staff must be specifically trained with respect to standard precautions to be taken for prevention and control of the infection. Standard precautions include hand and respiratory hygiene, the use of appropriate PPE based to risk assessment, environmental cleaning, biomedical 
Table 1. Highlights the important points to be considered for preparedness for a surgery during the COVID-19 pandemic

\section{Points} Yes/No

\section{Administrative support}

1. Is a robust and dedicated multidisciplinary team prepared?

2. Does the hospital have an infection control committee?

3. Is a written standard operating procedure (SOP) in place to implement all appropriate steps upon the notification of the first suspect case to fever OPD or Emergency room?

4. Are the staff members aware of the process for the safe movement of the suspect/confirmed COVID-19 patient through a defined pathway from the point of identification to the OR?

5. Are the personal protective equipment (PPE) (i.e. surgical masks, N95/FFP2/FFP3 respirators, gloves, gowns, goggles, face shields, and shoe covers) easily accessible to the health care staff?

6. Is the dedicated COVID operating area (close to the OR entrance) marked with a clearly labeled pathway?

7. Is the designated OR well ventilated?

Surgical Procedures

8. Is the surgery immediately required?

9. Is the nasopharyngeal and oropharyngeal sample collected from the suspected patient before the surgery?

10. Is the mode of surgery well planned by the operating team?

11. Are the items required in the OR prepared before the initiation of the procedure?

12. Does the operating team have the minimum number of people required, including the anesthetist?

13. Is the helping staff present in the anteroom for the delivery of items from anteroom if required? Infection prevention and control

14. Have all the staff members undergone the mandatory IPC training?

15. Does the hospital have a functional hand washing station (including water, soap, and paper towel)?

16. Are the alcohol-based hand rubs available in the OR?

17. Have posters illustrating donning/doffing and hand hygiene in local languages been put in the designated areas?

18. Are medical/surgical masks provided to all suspected and confirmed cases during transport?

19. Are health care staff trained on the rational use of N95/FFP2/FFP3 respirators (i.e., proper use and fit) and donning and doffing of the PPE?

\section{Environmental cleaning}

20. Are cleaning charts/checklists available for each area including the OR?

21. Are the cleaning staff members mopping from the least soiled area to the most soiled area and from high surfaces to low surfaces in the OR?

22. Are the high touch surfaces cleaned with $70 \%$ alcohol?

23. Are the low touch surfaces wet mopped with detergent followed by the use of $0.5 \%$ freshly prepared hypochlorite ensuring a contact time of 10 mins?

24. Is the OR cleaned and disinfected before the first procedure, between the procedures and after the last procedure (i.e., terminal cleaning)?

25. Is the corridors and elevators frequently cleaned ( 2 hourly)?

Biomedical waste Management

26. Is the SOP availability for BMW management?

27. Are there sufficient number of color-coded BMW bags available in the OR?

28. Are the bins labeled as 'Waste of COVID-19'

29. Is the biomedical waste discarded in the designated bin correctly?

30. Is the area dedicated for BMW collection available?

\section{Postoperative care}

31. Is the patient shifted directly to the isolation ICU/ward without going to the postoperative ward?

32. Are unnecessary visits minimized?

33. Are the discharge criteria fulfilled? 
waste, linens, and spill management, and sterilization of patient care equipment ${ }^{6}$. Distinct and clearly defined pathways must be prepared for shifting the patient from the emergency room to the operating room. Moreover, the patients that underwent an operation should not be kept in the same ward as the non-COVID patients.

\section{Operating Room (OR)}

All the COVID-19 cases must be operated in a designated OR. If available, negative pressure rooms should be preferred. The OR which is nearest to the theatre entrance area should be designated for COVID cases (Fig. 1). When multiple cases have to be operated, operating rooms nearer to the entrance in the particular theatre block must be reserved to curtail any environmental contamination. These rooms should be out of hightraffic areas and should not include non-essential materials. When an anteroom is available, this should be used as an area for donning (putting on) and doffing (taking off) of the PPE, exchange of equipment, medications, and any other materials required for the operation. However, it is emphasized that donning and doffing areas should be separate. Each OR should be properly ventilated with an integrated high-efficiency particulate air filter ${ }^{7}$. Instructional posters or signage on appropriate procedures should be prominently displayed. If an anteroom is not available, a taped off area should be marked for these activities just outside the OR door.

\section{Transfer of patient to the OR}

The patient should be transferred from the emergency room to the COVID operating room quickly. The respective OR staff and the team should be informed before shifting the patient to be prepared. Each hospital should map a well-defined short pathway using a few corridors and elevators for COVID patients. All designated allocations should be kept separate from other public areas in the hospital and, they have to be properly labeled to avoid confusion. Non-intubated patients must be given a 3-ply surgical mask. A dedicated ambulance should be used while shifting the patient to another hospital. The HCW should follow the basic infection control practices and wear recommended PPEs. In the ambulance, ideally, the patient's compartment should be kept separate from the driver's compartment. If the patient is shifted to the OR from any adjacent premises within the hospital, a stretcher can be used for transport. The HCW should wear appropriate PPEs (3-ply surgical mask, gloves, gown, and goggles) while shifting the patient and should minimize contact with the patient ${ }^{8}$. After transport, the stretcher should be

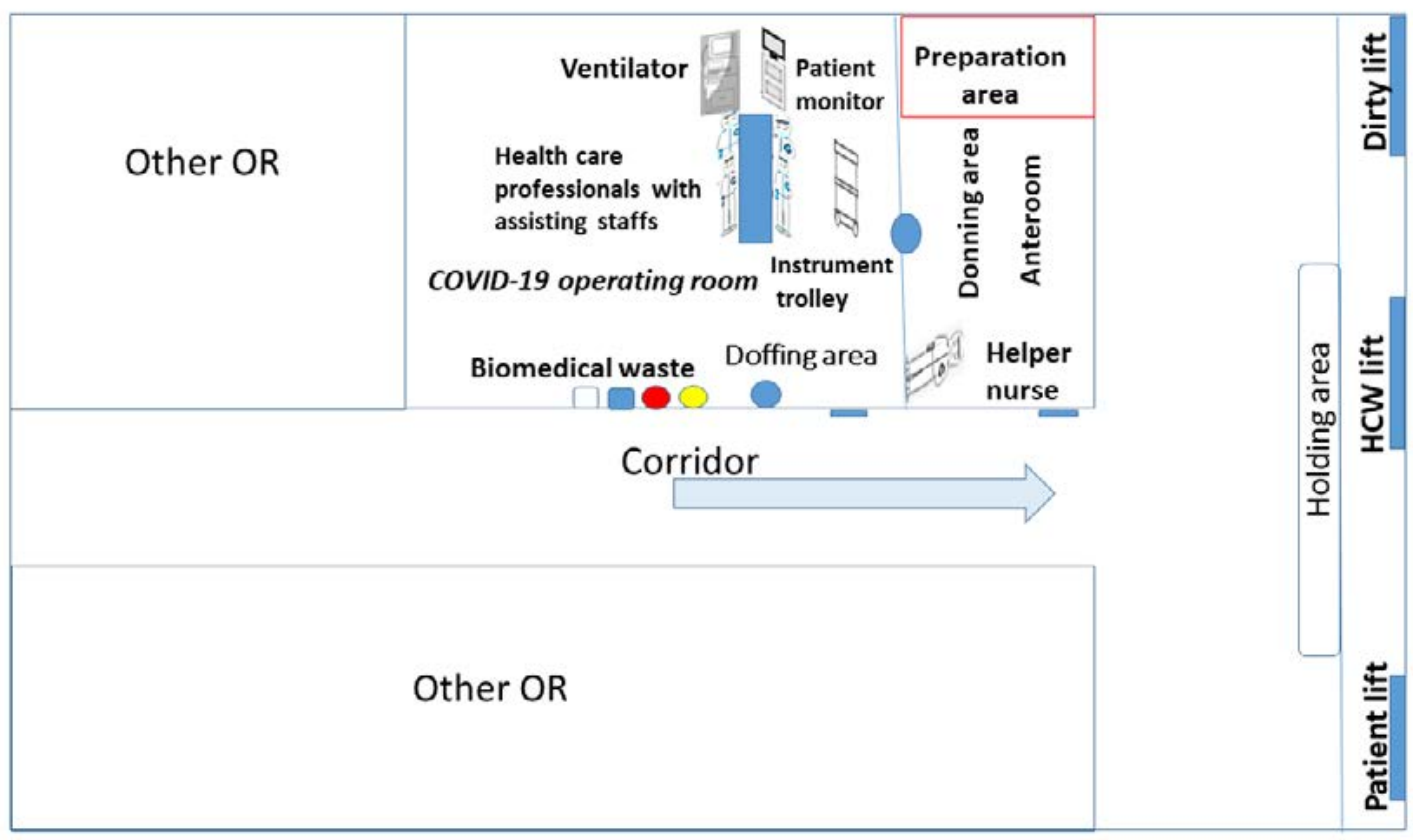

Fig. 1. The hospital operating area with the designated COVID-19 operating room and the anteroom. 
cleaned and disinfected followed by proper hand hygiene by the HCW. Utilized lifts must be cleaned and disinfected with $0.5 \%$ freshly prepared sodium hypochlorite solution ${ }^{9}$. The cleaning staff should be specifically trained concerning to infection control with special emphasis on environmental hygiene and the rational use of PPEs.

\section{Intraoperative management}

Only limited equipment based on the requirement of the procedure should be kept in the COVID OR. To minimize the risk of crossinfection, staff transiting should be minimized, and the available resources within the space should be utilized on a priority basis. Preoperative checklists with the addition of COVID-19 specific points should be filled thus helping in minimizing the risks of error. Coordination with the anesthesia team is extremely important. Standard dedicated anesthetic trolleys should be loaded with possible medications before initiating the procedure. All the AGPs such as intubation and extubation should be done with a limited number of experienced staff wearing complete PPE including the N95 respirator. Post anesthetic induction, including intubation, wait for 30 minutes of room ventilation thus reducing the viral bioburden, if possible. The rest of the team should then enter through the anteroom wearing complete PPE. A dedicated anesthesia machine with heat and moisture exchange filter and/or viral filters should be used. To reduce the risk of coughing, general anesthesia can be administered ${ }^{10}$. Non-intubated patients should be provided with a surgical mask inside the OR. Any sample collected for laboratory investigation must indicate the COVID-19 status of the patient (suspected/confirmed). All operating staff required in the procedure must not leave the OR until the complete procedure is carried out. Dedicated helping staff should be present in the anteroom, and when additional items are required in the $O R$, the helping staff should provide it through a medical trolley/pass box. Preferably, disposable materials should be used wherever possible including surgical items, linen, etc. Dedicated biomedical waste bins must be used to discard all disposable items. Reusable surgical instruments should be properly packed and transported to the central sterile supply department for cleaning, disinfection, and sterilization. Alcohol-based solutions for hand hygiene must always be available. After the procedure, when the patient leaves the OR, the next case must not be taken immediately in the same OR, thus giving time to reduce the viral bioburden. The air changes/hour of the operative room could be increased to $>25$ changes/hour for effectively reducing the viral load, whenever possible ${ }^{7}$. After the procedure, all areas must be thoroughly cleaned and disinfected. The patient should be reviewed, induced, and must recover within the OR itself, to restrict the contamination?

\section{Postoperative care}

Post-surgery the patient should be directly transferred to an isolation ICU/ward without shifting to the post-operative area via the designated elevator and corridor. The HCW shifting the patient from the OR should wear complete PPE and must provide a surgical mask to the patient. Visits to the patient should be minimized, especially those that are not necessary. Based on the patient's clinical improvement and at least two consecutive negative molecular results for SARSCoV-2, from the respiratory sample (collected at a gap of $24 \mathrm{~h}$ ), the previously confirmed case can be further shifted to the general ward or can be discharged and sent home ${ }^{11}$.

\section{Rational use of PPE}

Personal protection includes two important aspects of infection control i.e. wearing protective equipment and incorporating universal precautions. The PPE includes a face shield, pair of goggles, respirators (N95/FFP2/FFP3), fluidresistant gown, disposable latex gloves, and a shoe cover. The WHO recently published the PPE guidance for healthcare workers and emphasized on the rational use of PPE based on the risk assessment ${ }^{8}$. Surgical procedures in the ORs are included in the high-risk category. Moreover, special attention should be taken while donning the respirators (N95/FFP2/FFP3) to do a fit check. The HCW should strictly follow the procedures for donning and doffing the PPE and it must be done only in the designated areas. All the PPE must be considered contaminated while doffing, and therefore should be removed slowly without touching the outer surface. The PPE should be discarded into the designated biomedical waste bins followed by hand hygiene. 
Environmental cleaning and biomedical waste disposal

Studies have shown that the SARS CoV2 virus can survive on inanimate environmental surfaces for several hours to days ${ }^{12}$. Environmental cleaning is a part of the standard precautions and should be done meticulously throughout the day i.e. before the first procedure, between the procedures and after the last procedure (i.e. terminal cleaning). It is important to ensure that procedures involving cleaning and disinfecting the areas should be followed consistently. Thorough cleaning of contaminated surfaces with water and detergent followed by the use of U.S. Environmental Protection Administrationregistered hospital-level disinfectants such as $70 \%$ alcohol or freshly prepared $0.5 \%$ sodium hypochlorite (5000 ppm chlorine) solution ensuring a contact time of $10 \mathrm{~min}$ is effective. All horizontal, vertical, and contact surfaces should be cleaned and disinfected. High touch surfaces (OT table, light handle, infusion stands, monitors, and trolley handles) should be wiped with $70 \%$ alcohol while low touch surfaces (floor and walls) should be wet mopped with detergent followed by the use of disinfectants. Mopping should be done from the least soiled area to the most soiled area and from high surfaces to low surfaces ${ }^{9}$. Fogging or fumigation should not be done in the contaminated OR as it may create splashes that can further spread the virus. Potentially infected materials belonging to the patient should be disposed of properly in color-coded biomedical waste bins. Dedicated color-coded bins for biomedical waste should be kept inside the OR, to immediately discard all the contaminated items including the PPEs. Bins should be closed, sealed, and well-labeled as "waste of COVID 19" before being collected by the HCW. Sinks and taps should be cleaned by hand scrubbing them with detergent and water.

\section{CONCLUSION}

There is sufficient evidence that the best way to contain the spread of the pandemic is through the meticulous implementation of the IPC practices. On one hand, non-emergency procedures need to be delayed, and, at the same time all surgeries during this period should be proactively performed with complete universal precautions regardless of the COVID status of the patients. Prudent decisions and a conservative approach while making the best choices on surgeries will not only ensure personal protection but will also preserve the important resources, manpower, hospital beds, and equipment for repurposing in this urgent crisis. When the entire healthcare system is adapting to the rapid unforeseen changes caused due to the pandemic, strict adherence to the IPC practices along with a calm and vigilant approach appears to be the appropriate method for emergency surgeries in the current situation.

\section{ACKNOWLEDGMENTS}

None.

\section{CONFLICT OF INTEREST}

The authors declare that there is no conflict of interest.

\section{AUTHORS' CONTRIBUTION}

All listed author(s) have made a substantial, direct, and intellectual contribution to the work, and approved it for publication.

\section{FUNDING}

None.

\section{ETHICS STATEMENT}

This article does not contain any studies performed with human participants or animals.

\section{DATA AVAILABILITY}

All datasets generated or analyzed during this study are included in the manuscript and/or the Supplementary Files.

\section{REFERENCES}

1. Anita $P$, Jernigan $D B$. Initial public health response and interim clinical guidance for the 2019 novel coronavirus outbreak - United States, December 31, 2019-February 4, 2020. Morb Mortal Wkly Rep. 2020;69(5):140.

2. WHO. Naming the coronavirus disease (COVID-19) and the virus that causes it. World Health Organization 2020, Accessed 25 april, 2020.

3. WHO. Modes of transmission of virus causing COVID-19: implications for IPC precaution recommendations. Sci Br., 2020, Accessed 25 april, 2020.

4. Richard RW, Shimizu I. Hospital preparedness for emergency response: United States, 2008. Natl Health 
Stat Report. 2011;37:1-14.

5. Hua ZM, Boni L, Fingerhut A. Minimally Invasive Surgery and the Novel Coronavirus Outbreak: Lessons Learned in China and Italy. Ann Surg. 2020;272(1):e5e6. https://doi.org/10.1097/SLA.0000000000003924

6. John MA. Infection control: Its evolution to the current standard precautions. J Am Dent Assoc. 2003;134(5):569-574. https://doi.org/10.14219/jada. archive.2003.0222

7. Jolin W, Goh QY, Tan Z, et al. Preparing for a COVID-19 pandemic: a review of operating room outbreak response measures in a large tertiary hospital in Singapore. Can J Anesth. 2020;67:732-745. https:// doi.org/10.1007/s12630-020-01620-9

8. World Health Organization (WHO). Rational use of personal protective equipment for coronavirus disease (COVID-19) and considerations during severe shortages: interim guidance, 6 April 2020.WHO 2020; 1-7, Accessed 25 april, 2020.

9. $\quad \mathrm{CDC}$ and ICAN. Best Practices for Environmental
Cleaning in Healthcare Facilities in Resource-Limited Settings. Atlanta, GA: US Department of Health and Human Services, CDC; Cape Town, South Africa: Infection Control Africa Network; 2019.

10. Chen X, Liu Y, Gong Y, et al. Perioperative Management of Patients Infected with the Novel Coronavirus: Recommendation from the Joint Task Force of the Chinese Society of Anesthesiology and the Chinese Association of Anesthesiologists. Anesthesiology. 2020;132;1307-1316. https://doi.org/10.1097/ ALN.0000000000003301

11. Centers for Disease Control and Prevention (CDC). Discontinuation of Home Isolation for Persons with COVID-19 (Interim Guidance) 2020. https://www.cdc. gov/coronavirus/2019-ncov/hcp/disposition-in-homepatients.html, Accessed 25 april, 2020.

12. Alex WH Chin, Julie TS Chu, et al. Stability of SARSCoV-2 in different environmental conditions. The Lancet Microbe. 2020. https://doi.org/10.1016/s2666$5247(20) 30003-3$ 